\title{
Comparative characterization of SHED and DPSCs during extended cultivation in vitro
}

\author{
HUIHUI WANG ${ }^{1}$, QI ZHONG $^{2}$, TIANSHU YANG ${ }^{3}$, YING QI $^{1}$, MENGCHEN FU $^{1}$, \\ XI YANG ${ }^{1}$, LU QIAO $^{1}$, QI LING ${ }^{1}$, SHANGFENG LIU ${ }^{4}$ and YUMEI ZHAO ${ }^{1}$ \\ ${ }^{1}$ Shanghai Engineering Research Center of Tooth Restoration and Regeneration, Department of Pediatric Dentistry, School of \\ Stomatology, Tongji University, Shanghai 200072; ${ }^{2}$ Shanghai Ninth People's Hospital, Shanghai Jiaotong University, \\ Shanghai 200011; ${ }^{3}$ Shanghai Tenth People's Hospital, School of Medicine, Tongji University, Shanghai 200092; \\ ${ }^{4}$ Department of Stomatology, Huashan Hospital, Fudan University, Shanghai 200040, P.R. China
}

Received August 3, 2017; Accepted February 9, 2018

DOI: $10.3892 / \mathrm{mmr} .2018 .8725$

\begin{abstract}
Dental pulp stem cells (DPSCs) and stem cells from human exfoliated deciduous teeth (SHED) are types of human dental tissue-derived mesenchymal stem cells (MSCs). These cells possess a capacity for self-renewal, multilineage differentiation potential and immunomodulatory functions. Previous studies have reported that DPSCs and SHED may be beneficial in regenerative treatments and immunotherapy. The substantial expansion of cells in vitro is a prerequisite to obtaining adequate cell numbers required for cell-based therapy. However, the regeneration and clinical potential of MSCs diminishes with long-term cell culture amplification. To assess the alterations in SHED and DPSCs characteristics that underlie cellular senescence and result from extended in vitro amplification, the biological properties of SHED and DPSCs at passages 4 (P4) and 20 (P20) were compared. The cells underwent senescence following serial expansion to P20, as determined by altered cell morphology, decreased proliferation and migration capacity, attenuated differentiation potential, elevated senescence-associated $\beta$-galactosidase (SA- $\beta$-gal)-positive rates and increased apoptosis. The phenotypic changes were also accompanied by a marked increase in the expression of p53, p21 and p16 ${ }^{\text {Ink4a }}$. The
\end{abstract}

Correspondence to: Dr Yumei Zhao, Shanghai Engineering Research Center of Tooth Restoration and Regeneration, Department of Pediatric Dentistry, School of Stomatology, Tongji University, 339 Middle Yanchang Road, Shanghai 200072, P.R. China

E-mail: yumeizhao@tongji.edu.cn

Mr. Shangfeng Liu, Department of Stomatology, Huashan Hospital, Fudan University, 12 Urumqi Road, Shanghai 200040, P.R. China E-mail: shangfengliufudan@163.com

Abbreviations: DPSCs, dental pulp stem cells; SHED, stem cells from human exfoliated deciduous teeth; SA- $\beta$-gal, senescence-associated $\beta$-galactosidase; $\mathrm{P} 4$, passage 4; $\mathrm{P} 20$, passage 20

Key words: dental pulp stem cells, stem cells from human exfoliated deciduous teeth, biological characteristics, cellular senescence, in vitro cultivation present study also identified that senescent DPSCs exhibited an increased number of positive cells in SA- $\beta$-gal staining and demonstrated varying expressions of p53, p21 and p16 ${ }^{\text {Ink4a }}$ in comparison with SHED, indicating the involvement of diverse pathways in cellular senescence during long-term sequential in vitro culture and passage. Furthermore, at early and late passages, SHED exhibited a higher proliferation rate and osteogenic differentiation capability when compared with DPSCs. In addition, both cell types maintained their characteristic immunophenotype during long-term cultivation, while the expression levels of CD73 were higher in SHED at P20. The present study concluded that notable alterations were exhibited in SHED and DPSCs during the process of extensive expansion in vitro and the results may provide guidance for the selection of safe and effective expanded SHED and DPSCs for regenerative medicine and therapy.

\section{Introduction}

Mesenchymal stem cells (MSCs) are multipotent cells derived from the connective tissues of various organs, they serve critical roles in tissue regeneration and immunotherapy due to their self-renewal capacity, multilineage differentiation potential and immunosuppressive properties $(1,2)$. A previous study demonstrated that a particular subgroup of MSCs exists in human dental tissues, including dental pulp stem cells (DPSCs) and stem cells from human exfoliated deciduous teeth (SHED) (3).

DPSCs can be separated from enzymatically disaggregated adult human dental pulp tissue (4). Previous studies have revealed that DPSCs are highly clonogenic and rapidly proliferative, exhibit self-renewal and multiple differentiation capabilities (5), and have the potential for use in tissue regeneration and immunotherapy (6,7). SHED are derived from exfoliated deciduous teeth in the mixed dentition stages of children; they are a population of postnatal stem cells with the ability differentiate into various cell types (8). SHED are considered to be immature MSCs that are obtained from naturally exfoliated deciduous teeth, which may offer a unique, readily accessible and non-invasive stem cell resource with limited ethical and legal concerns $(9,10)$. Compared with DPSCs, SHED have been reported to exhibit a higher 
proliferation rate, differentiation potential and increased mineralization capacity in vivo, but also failed to regenerate a dentin-pulp-like complex $(8,11)$.

Based on the results of previous studies, researchers are increasingly inclined to select DPSCs and SHED as a source of stem cells for tissue regeneration and engineering, and for cellular-based therapies (6,7). In vitro expansion is necessary to obtain an adequate number of stem cells for use in tissue engineering and cell therapy strategies (12). However, MSCs exhibit certain alterations to their characteristics during long-term in vitro culture, including spontaneous malignant transformation, arrested growth, reduced differentiation capacity and shortened telomeres (13-15). Additionally, previous studies have indicated that the replicative senescence of MSCs is a continuous process during which MSCs exhibit an abnormal morphology, reduced expression of certain surface markers and arrested proliferation (16). These alterations affect the quality and efficacy of MSCs and ultimately hinder their practical application in clinical therapy. Thus, the analysis of SHED and DPSCs characteristics in in vitro cellular senescence is vital for basic research and quality control in cellular therapy. Additionally, the alterations to characteristics and the differences between SHED and DPSCs in long-term cultivation are yet to be elucidated.

The present study investigated the effects of long-term in vitro expansion on the basic properties and gene expression of SHED and DPSCs. The results revealed that their capacities for differentiation, proliferation and migration were decreased at passage $20(\mathrm{P} 20)$, in comparison with passage $4(\mathrm{P} 4)$. However, senescent SHED and DPSCs retained MSC-specific surface antigen markers. Furthermore, it was identified that the expression levels of certain markers associated with senescence were distinct at relatively advanced passages of SHED and DPSCs. The current study demonstrated that certain physiological and functional alterations occur during long-term culture and thus may provide guidance for the selection of suitable stem cells for therapeutic application, as well as insight into the various pathways of SHED and DPSCs in cellular senescence in vitro.

\section{Materials and methods}

Cell culture. Healthy and without dental caries exfoliated deciduous incisors were obtained from 30 children aged 6-7 years old and human impacted third molars were collected from 30 adults aged 18-25 years old (sex distribution of both groups, 1:1) from April 2015 to October 2015, all of whom had provided informed consent under the approved guidelines set by the Ethics Committee at the Affiliated Stomatology Hospital of Tongji University (Shanghai, China). Ethical approval was obtained from the Ethics Committee at the Affiliated Stomatology Hospital of Tongji University (approval no. 2015-010). Tooth surfaces were cleaned and the pulp tissue was gently separated from a remnant or from the mechanically fractured crown and root, following which the tissues were rinsed in PBS and minced into fragments of $0.5-1 \mathrm{~mm}^{3}$, which were subsequently uniformly placed in 6-well plates supplemented with Dulbecco's modified Eagle's medium (DMEM; cat. no. 11965-092; Gibco; Thermo Fisher Scientific, Inc., Waltham, MA, USA) containing 10\% fetal bovine serum
(FBS; cat. no. 16000044; Gibco; Thermo Fisher Scientific, Inc.), and $100 \mathrm{U} / \mathrm{ml}$ penicillin, $100 \mu \mathrm{g} / \mathrm{ml}$ streptomycin (cat. no. 15140-122; Gibco; Thermo Fisher Scientific, Inc.), as previously reported (17), and incubated at $37^{\circ} \mathrm{C}$ in $5 \% \mathrm{CO}_{2}$. The culture medium was replaced every 3 days and the cells were observed every $24 \mathrm{~h}$ using an inverted phase-contrast microscope, magnification, x100 (Nikon Corp., Tokyo, Japan). When $90 \%$ confluence was achieved, cells were harvested using $0.25 \%$ trypsin (Gibco; Thermo Fisher Scientific, Inc.), subcultured and conventionally cryopreserved in liquid nitrogen. Cells were cultured to P20 for subsequent experiments and each was repeated at least three times. Stem cells were collected from at least three different donors.

Flow cytometry analysis of cell surface antigens. SHED and DPSCs were harvested at P4 and P20, washed twice with PBS, passed through a $40 \mu \mathrm{m}$ cell strainer (BD Falcon; BD Biosciences, Franklin Lakes, NJ, USA) and then resuspended in PBS at a concentration of $1 \times 10^{7}$ cells $/ \mathrm{ml}$. The cells were subsequently incubated in the dark for $30 \mathrm{~min}$ on ice with various antibodies including: Anti-CD90-fluorescein isothiocyanate (FITC), anti-CD105-peridinin-chlorophyll-protein complex-cyanine 5.5, anti-CD73-allophycocyanin, anti-CD44-phycoerythrin (PE), anti-CD34-PE, anti-CD11b-PE, anti-CD19-PE, anti-CD45-PE and anti-human leukocyte antigen D-related (HLA-DR)-PE (all 1:50 dilution), according to the manufacturer's protocols. All of these antibodies were included in a Human MSC Analysis kit that was purchased from BD Biosciences (cat. no. 562245). Non-labeled cells and isotypes were used as negative controls. After being washed twice with PBS, the cells were analyzed with a flow cytometer (BD Biosciences) and a total of 10,000 events were acquired for each sample and data analysis was performed using FlowJo software version 7.6 (BD Biosciences).

Cell proliferation assay. P4 and P20 SHED and DPSCs were seeded into 96-well plates at a density of $3 \times 10^{3}$ cells/well with DMEM containing 10\% FBS and $100 \mathrm{U} / \mathrm{ml}$ penicillin, $100 \mu \mathrm{g} / \mathrm{ml}$ streptomycin, incubated at $37^{\circ} \mathrm{C}$ in $5 \% \mathrm{CO}_{2}$. Cell proliferation was determined using a Cell Counting kit- 8 assay performed at days $0,1,3$ and 5 , according to the manufacturer's protocol (cat. no. ZP328; Beijing Zoman Biotechnology Co., Ltd., Beijing, China).

In vitro differentiation. To evaluate cell differentiation, $\mathrm{P} 4 / 20$ SHED and DPSCs were seeded at a density of $4.2 \times 10^{3}$ cells $/ \mathrm{cm}^{2}$ (for osteogenic differentiation) and $2.1 \times 10^{4} \mathrm{cells} / \mathrm{cm}^{2}$ (for adipogenic differentiation) in 12/6-well plates with $\alpha$-minimum essential medium ( $\alpha$-MEM; R\&D Systems, Inc., Minneapolis, MN, USA) containing 10\% FBS and $100 \mathrm{U} / \mathrm{ml}$ penicillin, $100 \mu \mathrm{g} / \mathrm{ml}$ streptomycin. The cells were subsequently incubated overnight at $37^{\circ} \mathrm{C}$ and $5 \% \mathrm{CO}_{2}$. After reaching the $70 \%$ confluence (for osteogenic differentiation) and $100 \%$ confluence (for adipogenic differentiation), the medium was replaced with osteogenic or adipogenic differentiation medium to induce osteogenesis and adipogenesis. The osteogenic differentiation medium consistuted $\alpha$-MEM supplemented with $10 \% \mathrm{FBS}$ and $5 \%$ osteogenic supplement, and the adipogenic differentiation medium consisting of $\alpha$-MEM 
Table I. Primer sequences used in reverse transcription-quantitative polymerase chain reaction analysis.

\begin{tabular}{lll}
\hline Gene name & \multicolumn{1}{c}{ Forward primer sequences } & \multicolumn{1}{c}{ Reverse primer sequences } \\
\hline ALP & 5'-CCCAAGAATAAAACTGATGTG-3' & 5'-CTTCCAGGTGTCAACGAG-3' \\
Runx2 & 5'-GAATGCCTCTGCTGTTATG-3' & 5'-ACTCTTGCCTCGTCCACT-3' \\
PPAR $\gamma 2$ & 5'-GGTTGACACAGAGATGCC-3' & 5'-TGGAGTAGAAATGCTGGAGA-3' \\
p16Ink4a & 5'-ACTTCAGGGGTGCCACATTC-3' & 5'-CGACCCTGTCCCTCAAATCC-3' \\
p21 & 5'-GCGACTGTGATGCGCTAATG-3' & 5'-GAAGGTAGAGCTTGGGCAGG-3' \\
p53 & 5'-TGCTCAAGACTGGCGCTAAA-3' & 5'-CAATCCAGGGAAGCGTGTCA-3' \\
-actin & 5'-CTACCTCATGAAGATCCTCACCGA-3' & 5'-TTCTCCTTAATGTCACGCACGATT-3'
\end{tabular}

ALP, alkaline phosphatase; Runx2, runt-related transcription factor 2; PPAR $\gamma 2$, peroxisome proliferator-activated receptor $\gamma 2$.

supplemented with $10 \%$ FBS and $1 \%$ adipogenic supplement (cat. no. SC006; R\&D Systems, Inc.). The differentiation medium was replaced every 3 days. The cells were cultured for 14 days to induce adipogenic differentiation or for 21 days to induce osteogenic differentiation, following which the osteocytes, adipocytes and control group cells were harvested. The harvested cells were suspended in $1 \mathrm{ml}$ TRI Reagent ${ }^{\circledR}$ (Sigma-Aldrich; Merck KGaA, Darmstadt, Germany) for the detection of mRNA expression, and the cells were fixed in $4 \%$ paraformaldehyde for $30 \mathrm{~min}$ at room temperature, washed twice in PBS and stained with Alizarin Red or Oil Red O at room temperature for 30 min (Sigma-Aldrich; Merck KGaA), siained cells were observed using an inverted phase-contrast microscope (magnification, x100; Nikon Corporation, Tokyo, Japan), to assess the formation of mineralized nodules and the accumulation of lipid vacuoles, respectively. Stained Oil Red $\mathrm{O}$ was quantified by dissolving in $100 \%$ isopropanol and the optical density (OD) of the solution at $500 \mathrm{~nm}$ was measured by a microplate reader (Thermo Fisher Scientific, Inc.) (18). Stained Alizarin Red was extracted using $10 \%$ cetylpyridinium chloride buffer and the OD value of the solution was measured at $550 \mathrm{~nm}$ (19).

Reverse transcription-quantitative PCR (RT-qPCR). Total RNA was extracted from cells using TRI Reagent. cDNA was prepared using a TransScript First-Strand cDNA Synthesis SuperMix kit (Beijing TransGen Biotech Co., Ltd., Beijing, China). The RT reaction was set at an initial denaturation step at $42^{\circ} \mathrm{C}$ for $15 \mathrm{~min}$, followed by $85^{\circ} \mathrm{C}$ for $5 \mathrm{sec}$. Following first strand cDNA synthesis, qPCR was performed on an ABI Prism 7500 Sequence Detection System (Applied Biosystems; Thermo Fisher Scientific, Inc.) with a SuperReal PreMix Plus (SYBR-Green) kit (Tiangen Biotech Co., Ltd., Beijing, China). The reaction mixture was heated to $95^{\circ} \mathrm{C}$ for $15 \mathrm{~min}$, followed by amplification that consisted of 40 cycles of denaturation at $95^{\circ} \mathrm{C}$ for $10 \mathrm{sec}$, and annealing and extension at $60^{\circ} \mathrm{C}$ for $32 \mathrm{sec}$, according to the manufacturer's protocol. The relative alterations in gene expression were calculated using the $2^{-\Delta \Delta \mathrm{Cq}}$ method (20) with $\beta$-actin as a reference gene. The primer sequences that were utilized are listed in Table I.

Cell migration assay. A migration assay was conducted using Transwell inserts with an $8 \mu \mathrm{m}$ pore size (Corning Incorporated,
Corning, NY, USA) according to the manufacturer's protocol. Cells were seeded at a density of $2 \times 10^{4}$ cells/well into the upper chambers with serum-free DMEM while the lower chambers contained DMEM supplemented with 10\% FBS. Chambers were subsequently incubated for $24 \mathrm{~h}$ at $37^{\circ} \mathrm{C}$ and $5 \% \mathrm{CO}_{2}$. Migrated cells on the lower membranes were fixed with anhydrous methanol for $30 \mathrm{~min}$, wash the cells twice with PBS and stained with $0.1 \%$ crystal violet for $30 \mathrm{~min}$ at room temperature. They were then imaged using an upright fluorescent microscope (Nikon Corporation), magnification, x40 and counted in five independent, randomly selected fields of view.

Cell apoptosis assay. Apoptotic cells were detected using an Annexin V-FITC Apoptosis Detection kit (cat. no. ZP327; Beijing Zoman Biotechnology Co., Ltd.), according to the manufacturer's protocol. Cells were harvested and resuspended at a concentration of $1 \times 10^{6}$ cells $/ \mathrm{ml}$, the cell suspension (500 $\mu \mathrm{l}$ ) was centrifuged at $426 \mathrm{x}$ g for $5 \mathrm{~min}$ at room temperature, washed twice with cold $\mathrm{PBS}$, then resuspended in $500 \mu \mathrm{l}$ binding buffer, and incubated with FITC-conjugated Annexin V $(5 \mu \mathrm{l})$ and propidium iodide solution $(10 \mu \mathrm{l})$ for $15 \mathrm{~min}$ at room temperature in the dark, followed by flow cytometer analysis and data analysis was performed using FlowJo software version 7.6 (BD Biosciences).

Senescence-associated $\beta$-galactosidase (SA- $\beta$-gal) assay. Cellular senescence was assessed via SA- $\beta$-gal staining (21), which was performed using the Senescence Cells Histochemical Staining kit (cat. no. CS0030; Sigma-Aldrich; Merck KGaA), according to the manufacturer's protocol. Cells were seeded into 12 -well plates at a density of $2 \times 10^{5}$ cells/well and cultured in DMEM containing 10\% FBS and $100 \mathrm{U} / \mathrm{ml}$ penicillin, $100 \mu \mathrm{g} / \mathrm{ml}$ streptomycin. Positive staining was evaluated following overnight incubation at $37^{\circ} \mathrm{C}$ in a $\mathrm{CO}_{2}$-free atmosphere. The number of blue-stained cells and the total number of cells were counted from five distinct fields under a phase-contrast microscope and subsequently calculate the percentage of blue-stained cells (senescent cells) in the total number of counted cells.

Western blot analysis. SHED and DPSCs were collected and lysed with RIPA lysis buffer containing phosphatase and protease inhibitor cocktail (Beyotime Institute of 
A

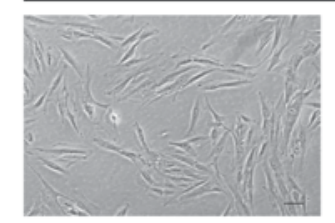

SHED

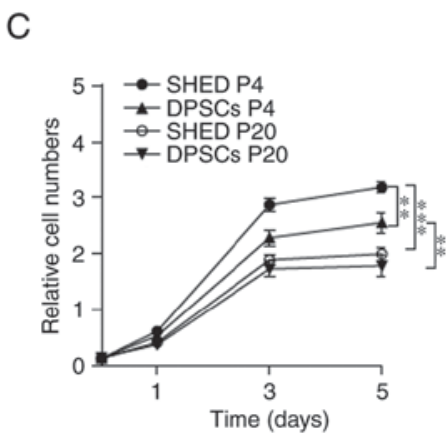

P4

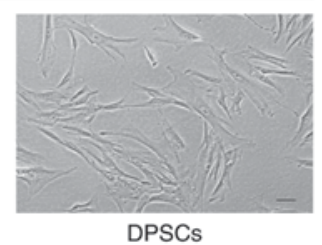

D

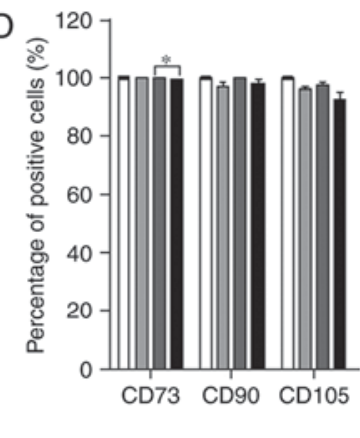

B

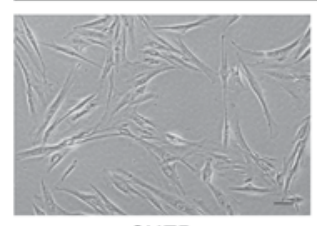

SHED

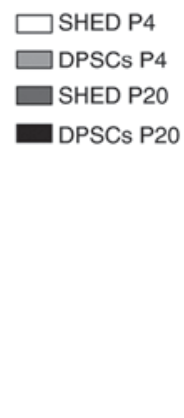

E
P20
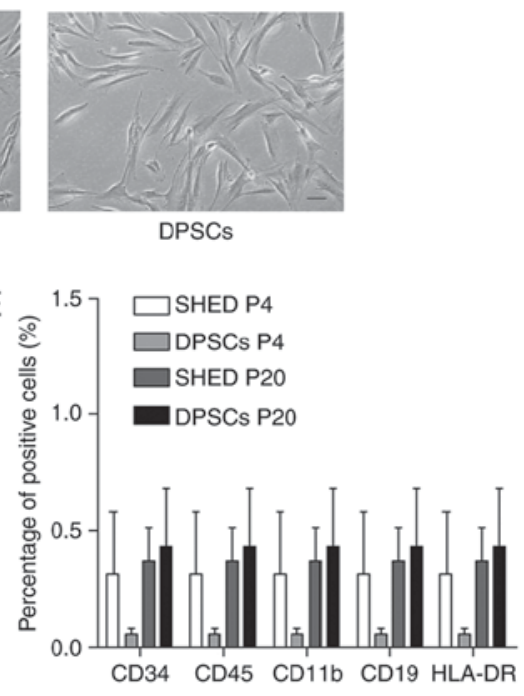

Figure 1. SHED and DPSCs morphology, proliferation and immunophenotype. Representative morphological features of SHED and DPSCs at (A) P4 and (B) P20. Scale bars, $100 \mu \mathrm{m}$. (C) Proliferation curves of SHED and DPSCs at P4 and P20. (D) Statistical analysis of the percentage of CD73-, CD90- or CD105-positive cells in SHED and DPSCs groups at P4 and P20. (E) Statistical analysis of the percentage of CD34-, CD11b-, CD19-, CD45- and HLA-DR-positive cells of the SHED and DPSCs groups at P4 and P20. Data are presented as the mean \pm standard error of the mean $(n=3)$. ${ }^{*} \mathrm{P}<0.05$, ${ }^{* *} \mathrm{P}<0.01$ and ${ }^{* * *} \mathrm{P}<0.001$, as indicated. SHED, stem cells from human exfoliated deciduous teeth; DPSCs, dental pulp stem cells; P4/20, passage 4/20; HLA-DR, human leukocyte antigen D-related.

Biotechnology, Beijing, China). Protein concentrations were determined by the Bicinchoninic Acid protein assay kit (Pierce; Thermo Fisher Scientific, Inc.). Total proteins $(20 \mu \mathrm{g})$ were separated using 10\% SDS-PAGE and transferred to nitrocellulose membranes. Membranes were subsequently blocked with $5 \%$ skimmed milk powder in PBS-Tween-20 (PBST; $0.1 \%$ Tween-20) for $2 \mathrm{~h}$ at room temperature and gently agitated with the following primary antibodies overnight at $4^{\circ} \mathrm{C}$ : Rabbit anti-p16 ${ }^{\text {Ink4a }}$ (cat. no. 10883-1-AP; dilution, 1:1,000; ProteinTech Group, Inc., Chicago, IL, USA), rabbit anti-p21 (cat. no. 10355-1-AP; dilution, 1:1,000; ProteinTech Group, Inc.), rabbit anti-p53 (cat. no. 10442-1-AP; dilution, 1:2,000; ProteinTech Group, Inc.) and rabbit anti-GAPDH (cat. no. G9545; dilution, 1:10,000; Sigma-Aldrich; Merck KGaA). Subsequently, the membranes were washed in PBST and incubated with goat anti-rabbit horseradish peroxidase-conjugated secondary antibodies (cat. no. 31460; dilution, 1:10,000; Invitrogen; Thermo Fisher Scientific, Inc.) for $1 \mathrm{~h}$ at room temperature. Proteins were detected using an ECL detection system (Pierce; Thermo Fisher Scientific, Inc.).

Statistical analysis. All the results were performed at least 3 times and data are presented as the mean \pm standard error of the mean. All statistical analyses were conducted using SPSS software version 20.0 (IBM Corp., Armonk, NY, USA). Comparisons of normally distributed data were performed using one-way analysis of variance followed by Tukey's post-hoc test (for multiple comparisons) or Student's t-tests (for two samples). Group comparisons of skewed distributed data were analyzed using the Kruskal-Wallis $\mathrm{H}$ test and variations of statistical significance between groups were further subjected to post-hoc pairwise analysis by applying Dunn's-Bonferroni tests. $\mathrm{P}<0.05$ was considered to indicate a statistically significant difference.

\section{Results}

Morphology and long-term growth kinetics of SHED and DPSCs. Following isolation, the majority of SHED and DPSCs exhibited a spindle shape, small size and low granularity during continued culture to P4 (Fig. 1A). Long-term growth (to P20) led to previously observed (in P4) and typical morphological alterations, as SHED and DPSCs were notably larger with irregular and elongated shapes (Fig. 1B). SHED exhibited a significantly higher proliferation rate in comparison with DPSCs in vitro at an early passage (P4). However, the rapid growth kinetics of SHED cells significantly decreased by P20 and no differences were identified between SHED and DPSCs at P20 (Fig. 1C). These results indicate that SHED and DPSCs lose their proliferative potential with consecutive cell passaging.

Immunophenotype of SHED and DPSCs. To assess the effects of continuous expansion on the immunophenotype of SHED and DPSCs, stem cell surface antigens were assessed using flow cytometry. The results demonstrated that cells maintained the characteristic immunophenotype of MSCs, with high rates of positive CD73, CD90 and CD105 expression, and low expression of CD34, CD11b, CD19, CD45 and HLA-DR during long-term expansion (Fig. 1D and E). However, the percentage of CD73-positive DPSCs was lower at P20, in comparison with SHED at P20 (Fig. 1D). These results indicated that SHED and DPSCs were able to maintain their specific immunophenotypes during long-term expansion and that SHED exhibited more properties of stem cells.

Biological characteristics of SHED and DPSCs. The present study investigated whether the differentiation potential of cells was influenced by long-term cultivation in vitro. Oil Red O staining demonstrated that the presence of lipid vesicles was 

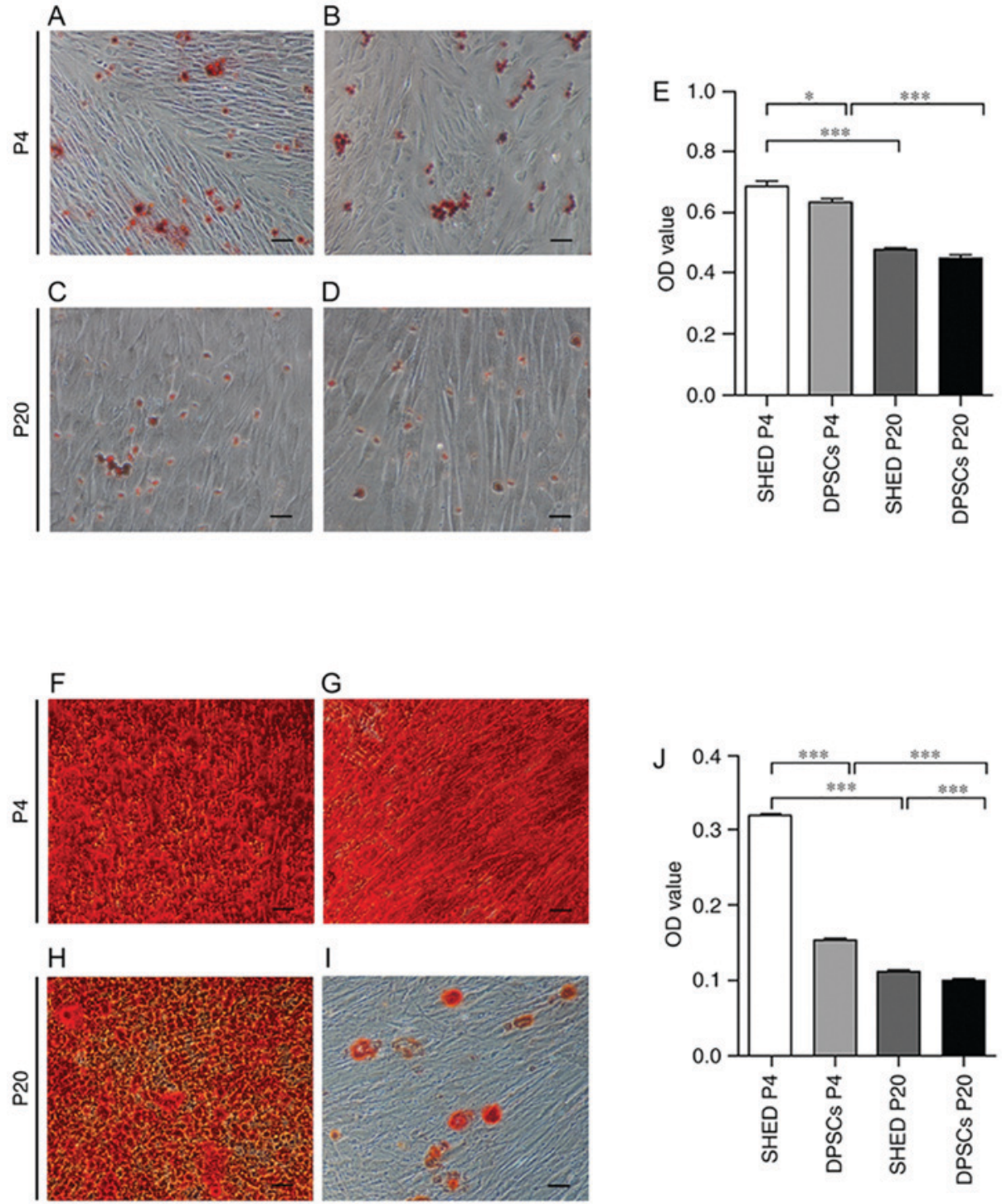

Figure 2. In vitro differentiation of SHED and DPSCs. Adipogenesis was assessed by Oil Red O staining of (A) SHED at P4, (B) DPSCs at P4, (C) SHED at P20 and (D) DPSCs at P20. Scale bars, $100 \mu \mathrm{m}$. (E) Oil Red O staining quantification of adipogenic differentiation of SHED and DPSCs at P4 and P20). Osteogenesis was assessed by Alizarin Red staining of (F) SHED at P4, (G) DPSCs at P4, (H) SHED at P20 and (I) DPSCs at P20. Scale bars, $100 \mu \mathrm{m}$. (J) Alizarin Red staining quantification of osteogenic differentiation of SHED and DPSCs at P4 and P20. Data are presented as the mean \pm standard error of the mean $(n=4) .{ }^{*} \mathrm{P}<0.05$ and ${ }^{* * *} \mathrm{P}<0.001$, as indicated. SHED, stem cells from human exfoliated deciduous teeth; DPSCs, dental pulp stem cells; $\mathrm{P} 4 / 20$, passage 4/20; OD, optical density.
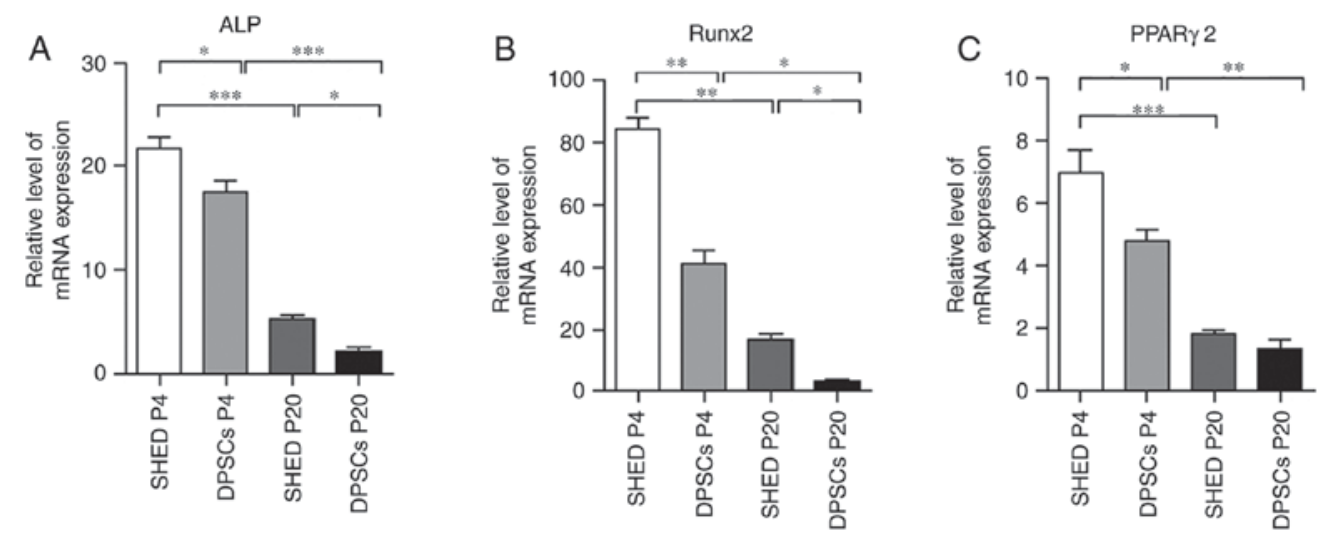

Figure 3. Quantification of osteogenic and adipogenic markers. Reverse transcription-quantitative polymerase chain reaction was performed to determine the mRNA expression levels of (A) ALP, (B) Runx2 and (C) PPAR $\gamma 2$ in SHED and DPSCs at P4 and P20 following in vitro differentiation. ALP and Runx2 were considered to be osteogenic markers, while PPAR $\gamma 2$ was considered to indicate adipogenic differentiation. Data are presented as the mean \pm standard error of the mean $(n=3) .{ }^{*} \mathrm{P}<0.05,{ }^{* *} \mathrm{P}<0.01$ and ${ }^{* * *} \mathrm{P}<0.001$, as indicated. ALP, alkaline phosphatase; Runx 2, runt-related transcription factor 2; PPAR $\gamma 2$, peroxisome proliferator-activated receptor $\gamma 2$; SHED, stem cells from human exfoliated deciduous teeth; DPSCs, dental pulp stem cells; P4/20, passage 4/20. 
A

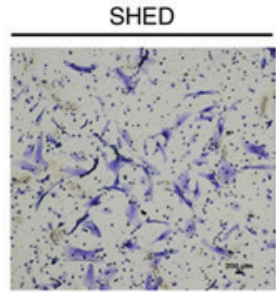

DPSCs

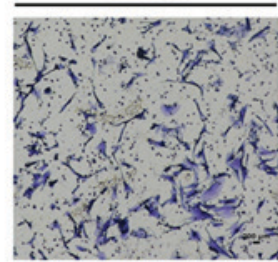

B
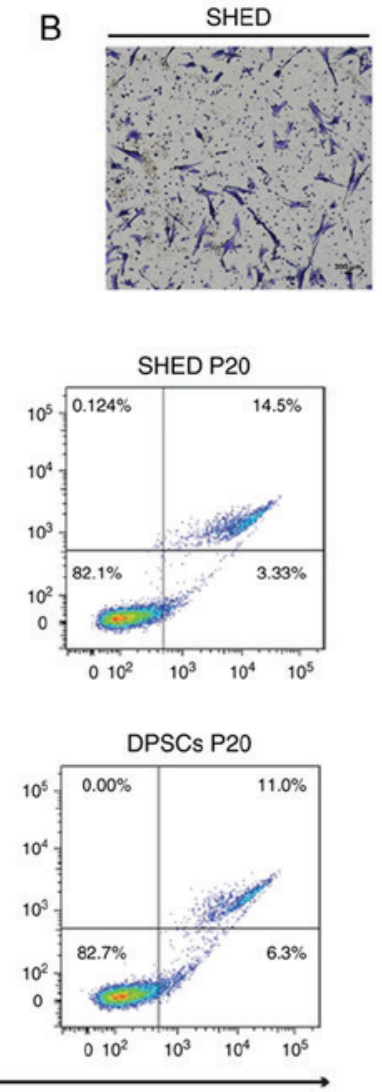

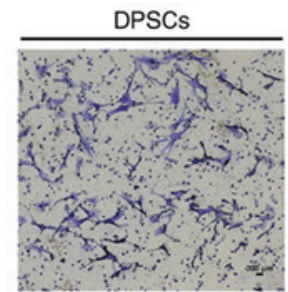

$\mathrm{E}$

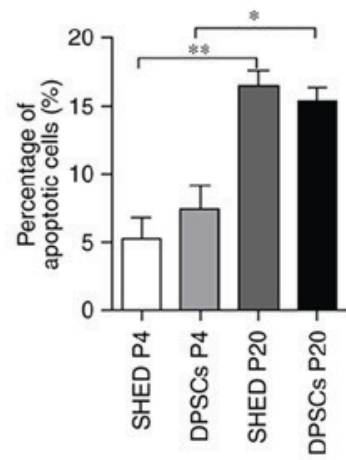

Figure 4. Senescence-associated alterations in SHED and DPSCs during prolonged culture in vitro. Crystal violet staining revealed the migratory cells of SHED and DPSCs at (A) P4 and (B) P20. Scale bars, $200 \mu \mathrm{m}$. (C) Quantitative analysis of migratory cells in each group. (D) Representative flow cytometry plots indicating the apoptotic cell proportions of SHED and DPSCs groups (upper and lower right-hand side quadrants combined). (E) Statistical analysis of the apoptotic cell proportions of SHED and DPSCs groups. Data are presented as the mean \pm standard error of the mean $(\mathrm{n}=3)$. ${ }^{*} \mathrm{P}<0.05$ and ${ }^{* *} \mathrm{P}<0.01$, as indicated. SHED, stem cells from human exfoliated deciduous teeth; DPSCs, dental pulp stem cells; P4/20, passage 4/20; FITC, fluorescein isothiocyanate.

higher in SHED compared with DPSCs at P4 and that the adipogenic differentiation potential was decreased in SHED and DPSCs at P20 in comparison with at P4. However, no marked differences were observed between the two groups following consecutive passage in vitro (Fig. 2A-E). The Alizarin Red-positive condensed nodules of SHED at an early passage were larger and denser compared with DPSCs. At a later passage, DPSCs still exhibited reduced formations of mineralized nodules compared with SHED, but Alizarin Red staining in SHED cells at P20 was also significantly reduced compared with SHED cells at P4 (Fig. 2F-J). Therefore, the propensity for adipogenic and osteogenic differentiation in DPSCs and SHED cells was attenuated with long-term cultivation. Furthermore, RT-qPCR analysis of differentiation-associated gene expression levels, including the osteoblast marker genes alkaline phosphatase and runt-related transcription factor 2, and the adipocyte-specific transcript, peroxisome proliferator-activated receptor $\gamma 2$, verified this conclusion; mRNA expression levels of these markers were all reduced in DPSCs and SHED cells at P20 compared with P4 (Fig. 3).

The motility of SHED and DPSCs was subsequently assessed. It was demonstrated that the migratory abilities of DPSCs and SHED cells significantly decreased with an increase in passage number (Fig. 4A-C). However, the quantification of migratory cell numbers exhibited no marked difference between SHED and DPSCs at the same passage number (Fig. 4A-C). Cellular senescence is associated with DNA damage accumulation and usually leads to cell apoptosis (22). The present study therefore assessed the number of apoptotic cells using flow cytometry. The results demonstrated that long-term cultivation was associated with a progressive increase in the number of apoptotic cells in both DPSCs and SHED cells (Fig. 4D and E). Furthermore, the percentage of apoptotic cells in the DPSC group was similar to that in the SHED group, irrespective of passage number (Fig. 4D and E).

Expression of senescence molecular markers in vitro. To determine whether the long-term culture of cells to P20 caused replicative senescence in SHED and DPSCs, P4/20 SHED and DPSCs were stained with SA- $\beta$-gal, a verified cell senescence marker (23). The results demonstrated that long-term culture induced marked SA- $\beta$-gal expression in SHED and DPSCs (Fig. $5 \mathrm{~A}$ and B). The percentage of SA- $\beta$-gal-positive SHED significantly increased with passage number, from $1.31 \pm 0.25 \%$ at $\mathrm{P} 4$ to $13.44 \pm 2.29 \%$ at P20, while in DPSCs, SA- $\beta$-gal activity significantly increased from $6.96 \pm 1.02$ to $25.62 \pm 1.10 \%$ at P4 and P20, respectively (Fig. 5C). The results indicated that DPSCs exhibited a higher predisposition towards senescence during long-term culture, compared with SHED.

The expression of typical senescence markers p53, p21 and $16^{\text {Ink4a }}$ in SHED and DPSCs at each passage number 
A

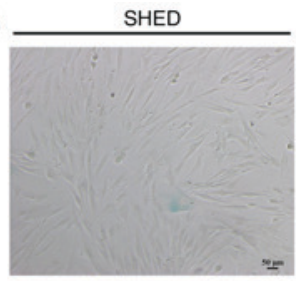

DPSCs

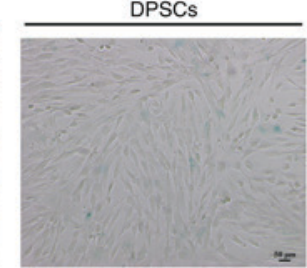

B

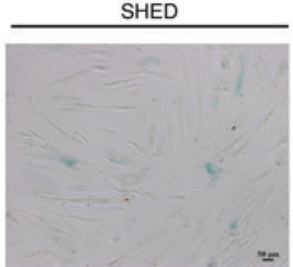

DPSCs

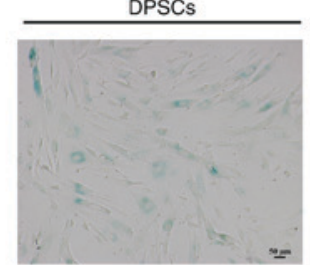

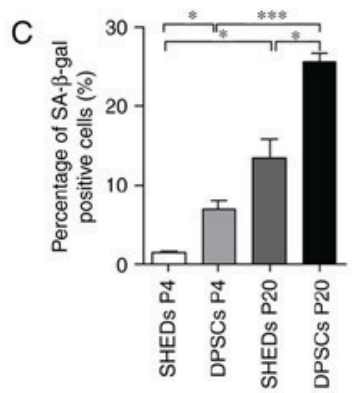
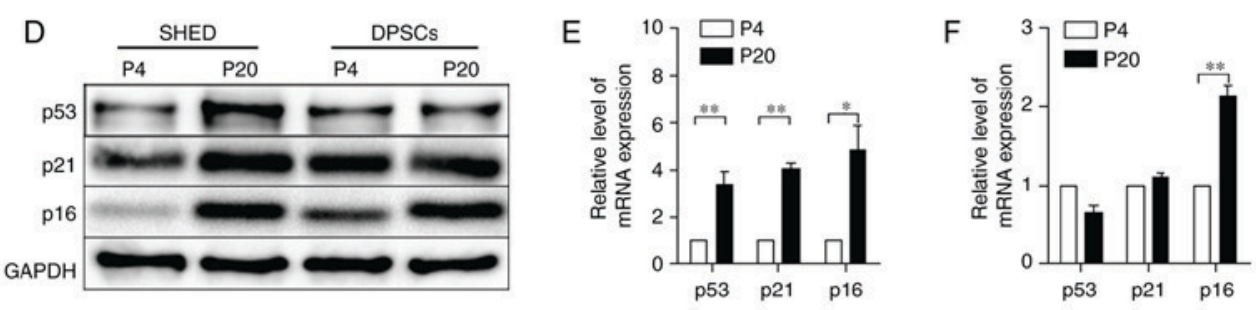

Figure 5. Phenotypic and molecular characterizations of SHED and DPSCs in senescence. Representative images of SA- $\beta$-gal-positive cells in SHED and DPSCs at (A) P4 and (B) P20. Scale bar, $50 \mu \mathrm{m}$. (C) The percentage of SA- $\beta$-gal-positive cells in SHED and DPSCs. (D) Western blot analysis of p53, p21 and $16^{\text {Ink4a }}$ expression in SHED and DPSCs. Reverse transcription-quantitative polymerase chain reaction analysis of p53, p21 and p16 ${ }^{\text {Ink4a }}$ mRNA expression normalized to GAPDH in (E) SHED and (F) DPSCs at P4 and P20. Data are presented as the mean + standard error of the mean $(\mathrm{n}=3)$. ${ }^{*} \mathrm{P}<0.05,{ }^{* *} \mathrm{P}<0.01$ and ${ }^{* * *} \mathrm{P}<0.001$, as indicated. SHED, stem cells from human exfoliated deciduous teeth; DPSCs, dental pulp stem cells; SA- $\beta$-gal, senescence-associated $\beta$-galactosidase; $\mathrm{P} 4 / 20$, passage $4 / 20$.

were subsequently assessed to confirm these results at the mRNA and protein level. The results showed that consecutive passaging led to a marked increase in the mRNA and protein expression of p53, p21 and p16 ${ }^{\text {Ink4a }}$ in SHED cells (Fig. 5D-F). However, the mRNA and protein levels of p53 and p21 were not significantly different between P4 and P20 in DPSCs, while the expression of the senescence marker p16 ${ }^{\text {Ink4a }}$ was significantly increased by P20 (Fig. 5D-F). These results demonstrated that SHED and DPSCs may undergo distinct pathways during cellular senescence and that $\mathrm{p} 16^{\text {Ink4a }}$ appears to serve a more prominent role in the senescence of DPSCs.

\section{Discussion}

DPSCs and SHED have potential advantages for tissue regenerativeengineering and therapeuticapplicationsdueto their multilineage differentiation potential and immunomodulatory properties (3). Cell therapy protocols require 10-400 million human MSCs per treatment and consequently, MSCs must be expanded in vitro prior to clinical application (24). Over five decades ago, Hayflick (25) demonstrated that all primary human cells exhibit a finite proliferative capacity in culture and, following a limited number of cell divisions, enter cellular senescence. Further studies have reported that MSCs undergo considerable alterations during in vitro expansion, including spontaneous transformation, reduced capacity for trafficking and homing, epigenetic and gene expression alterations, and morphological and multipotent differentiation potential alterations (26-28). However, no marked indications of culture degeneration or spontaneous differentiation have been demonstrated in SHED during long-term culture up to P19 (29). Thus, it remains unclear whether SHED and DPSCs undergo considerable characteristic alterations during in vitro culture up to P20. In particular, the molecular mechanisms underlying the phenotypical changes in SHED and DPSCs during culture expansion remain poorly understood.

A previous study demonstrated that SHED and DPSCs at early passage numbers exhibited differences in growth and differentiation (11). However, differences in their characteristics and gene expression profiles at later passage numbers are yet to be elucidated. The present study cultured primary SHED and DPSCs and consecutively expanded them in vitro. It was demonstrated that SHED exhibited a significantly higher proliferation rate, compared with DPSCs at P4, which is similar to the results of a previous study (11). At P20, both SHED and DPSCs exhibited a progressive loss of proliferative capacity and morphological alterations, despite maintaining their specific immunophenotype, as previously observed in other types of MSCs, such as bone marrow MSC (30). DPSCs became larger in size, with a more granulated cytoplasm. In addition, marginal decreases in the expression levels of CD73 were exhibited following long-term sequential cell passaging in vitro in DPSCs compared with SHED.

A previous study demonstrated that human MSCs exhibited reduced differentiation potential as a result of prolonged culture in vitro (15). However, a previous study also demonstrated that adipogenic differentiation potential decreases at later passages, whereas the propensity for osteogenic differentiation increases in replicative senescence, suggesting that long-term culture impacts the differentiation potential of MSCs (16). The present study indicated that the adipogenic and osteogenic differentiation potential was reduced with an extended expansion period and that the two groups exhibited different capacities for differentiation. This may therefore provide guidance for the selection of cell sources for tissue regenerative medicine. A previous study also demonstrated that MSCs, which exhibit higher cell migratory capacities, are more appropriate for tissue regeneration in terms of cell homing ability (31). The current study therefore 
compared the migratory ability of SHED and DPSCs during prolonged in vitro expansion. The results demonstrated that cell migratory capacity was decreased at P20 compared with P4 in each group. However, no significant differences were identified between the cell migratory abilities of SHED and DPSCs at early or at later passage stages. In addition, SHED showed a higher proliferation rate and differentiation capability compared with in DPSCs in vitro, consequently, these results indicate that SHED may be an accessible, suitable and potential alternative source for regenerative medicine and therapeutic application.

Cell senescence is an irreversible process that is typically accompanied by the upregulation of senescence regulators, including $\mathrm{p} 53 / \mathrm{p} 21$ and $\mathrm{p} 16^{\text {Ink4a }}$, and the increased activity of SA- $\beta$-gal (32). The results of the present study indicated that the percentage of SA- $\beta$-gal positive SHED and DPSCs increased with passage number. At P20, the number of SA- $\beta$-gal-positive SHED was reduced in comparison with DPSCs, indicating that a higher number of senescent cells were detected in DPSCs under standard culture conditions during long-term expansion, compared with SHED cells. However, no significant differences were identified in the number of apoptotic cells between the two groups at P20. This counterintuitive observation indicated that DPSCs may initiate an adaptive response to adopt a compromised state to avoid being eliminated during severe cellular senescence in vitro. This phenomenon was similar to that exhibited by neural stem cells (23). p53, p21 and/or p16 ${ }^{\text {Ink4a }}$ are key molecules that contribute to cell cycle arrest and cellular senescence (33). The current study assessed the expression of $\mathrm{p} 53, \mathrm{p} 21$ and $\mathrm{p} 16^{\text {Ink4a }}$ at each passage number in the two groups. The results demonstrated that the mRNA and protein expression of $\mathrm{p} 53, \mathrm{p} 21$ and $\mathrm{p} 16^{\text {Ink4a }}$ markedly increased at later passage numbers in SHED, while only p16 ${ }^{\text {Ink4a }}$ was significantly increased in DPSCs at a later passage, with a decrease observed for p53 and only a marginal increase in p21 levels at a later passage in DPSCs. The reason for this observation may be that as the number of growth-arrested cells increases with passage, most cells become senescent and culture growth flattens, leading to decrease in the number of DPSCs at P20. In addition, DPSCs may adapt a compromised state to prevent persistent DNA damage, indeed, similar observations have been noted in a previous study (24). These distinctions indicated that various distinct pathways may be involved in SHED and DPSCs cellular senescence during long-term culture in vitro and that $\mathrm{p} 16^{\mathrm{Ink} 4 \mathrm{a}}$ may be a primary gene associated with the cellular senescence of DPSCs.

The mechanisms of SHED and DPSCs entry into senescence during long-term culture in vitro remain inadequately understood. A previous study demonstrated that telomeric DNA is subjected to gradual erosion following the accumulation of MSCs due to consecutive expansion (34). Telomere erosion leads to the exposure of uncapped, free double-stranded chromosome ends, which initiates a persistent DNA damage response, and under these conditions, the recruitment of ataxia telangiectasia mutated, which is a damage sensor to uncapped telomeres, subsequently resulting in the stabilization of tumor suppressor $\mathrm{p} 53$, enhanced expression of the $\mathrm{p} 53$ transcriptional target, $\mathrm{p} 21$, or activated $\mathrm{p} 16^{\text {Ink4a }}(22)$. $\mathrm{p}^{\text {Ink4a }}$ has been reported to block the CDK4- and CDK6-mediated inactivation of retinoblastoma to prevent cell cycle progression and drive cell entry into senescence (32-34). Further studies investigating the mechanism involved in the in vitro cellular senescence of SHED and DPSCs are therefore required.

In conclusion, the present study indicated that SHED and DPSCs exhibit distinct biological characteristics and gene expression profiles, and different pathways may be activated in each of these cell types during long-term in vitro cultivation. Furthermore, the results provide insight into the appropriate selection of passaged SHED and DPSCs for use in cell-based therapies.

\section{Acknowledgements}

Not applicable.

\section{Funding}

The present study was supported by the Science and Technology Commission of Shanghai (grant no. 14411963600).

\section{Availability of data and materials}

The datasets used and/or analyzed during the current study are available from the corresponding author on reasonable request.

\section{Authors' contributions}

HW contributed to the conception and design, collection and assembly of data, data analysis and interpretation and writing of the manuscript. QZ, TY, YQ, MF, XY, LQ and QL contributed to the study design, data analysis and interpretation. YZ and SL participated in the conception and design of the study, data analysis and interpretation, financial acquisition, manuscript writing and final approval of manuscript. All authors read and approved the final manuscript.

\section{Ethics approval and consent to participate}

Ethical approval was obtained from the Ethics Committee at the Affiliated Stomatology Hospital of Tongji University (approval no. 2015-010). All individuals that participated in the study provided informed consent under the approved guidelines set by the Ethics Committee at the Affiliated Stomatology Hospital of Tongji University.

\section{Consent for publication}

Not applicable.

\section{Competing interests}

The authors declare that they have no competing interests.

\section{References}

1. Salem HK and Thiemermann C: Mesenchymal stromal cells: Current understanding and clinical status. Stem Cells 28: 585-596, 2010.

2. Reddy BY, Xu DS and Hantash BM: Mesenchymal stem cells as immunomodulator therapies for immune-mediated systemic dermatoses. Stem Cells Dev 21: 352-362, 2012. 
3. Liu J, Yu F, Sun Y, Jiang B, Zhang W, Yang J, Xu GT, Liang A and Liu S: Concise reviews: Characteristics and potential applications of human dental tissue-derived mesenchymal stem cells. Stem Cells 33: 627-638, 2015.

4. Gronthos S, Mankani M, Brahim J, Robey PG and Shi S: Postnatal human dental pulp stem cells (DPSCs) in vitro and in vivo. Proc Natl Acad Sci USA 97: 13625-13630, 2000.

5. Gronthos S, Brahim J, Li W, Fisher LW, Cherman N, Boyde A, DenBesten P, Robey PG and Shi S: Stem cell properties of human dental pulp stem cells. J Dent Res 81: 531-535, 2002.

6. Bakopoulou A and About I: Stem cells of dental origin: Current research trends and key milestones towards clinical application. Stem Cells Int 2016: 4209891, 2016.

7. Li Z, Jiang CM, An S, Cheng Q, Huang YF, Wang YT, Gou YC, Xiao L, Yu WJ and Wang J: Immunomodulatory properties of dental tissue-derived mesenchymal stem cells. Oral Dis 20: 25-34, 2014.

8. Miura M, Gronthos S, Zhao M, Lu B, Fisher LW, Robey PG and Shi S: SHED: Stem cells from human exfoliated deciduous teeth. Proc Natl Acad Sci USA 100: 5807-5812, 2003.

9. Kashyap R: SHED-basic structure for stem cell research. J Clin Diagn Res 9: ZE07-ZE09, 2015.

10. Brar GS and Toor RS: Dental stem cells: Dentinogenic, osteogenic, and neurogenic differentiation and its clinical cell based therapies. Indian J Dent Res 23: 393-397, 2012.

11. Wang X, Sha XJ, Li GH, Yang FS, Ji K, Wen LY, Liu SY, Chen L, Ding Y and Xuan K: Comparative characterization of stem cells from human exfoliated deciduous teeth and dental pulp stem cells. Arch Oral Biol 57: 1231-1240, 2012.

12. Banfi A, Muraglia A, Dozin B, Mastrogiacomo M, Cancedda R and Quarto R: Proliferation kinetics and differentiation potential of ex vivo expanded human bone marrow stromal cells: Implications for their use in cell therapy. Exp Hematol 28: 707-715, 2000.

13. Røsland GV, Svendsen A, Torsvik A, Sobala E, McCormack E, Immervoll $\mathrm{H}$, Mysliwietz J, Tonn JC, Goldbrunner R, Lønning PE, et al: Long-term cultures of bone marrow-derived human mesenchymal stem cells frequently undergo spontaneous malignant transformation. Cancer Res 69: 5331-5339, 2009.

14. Baxter MA, Wynn RF, Jowitt SN, Wraith JE, Fairbairn LJ and Bellantuono I: Study of telomere length reveals rapid aging of human marrow stromal cells following in vitro expansion. Stem Cells 22: 675-682, 2004.

15. Bonab MM, Alimoghaddam K, Talebian F, Ghaffari SH, Ghavamzadeh A and Nikbin B: Aging of mesenchymal stem cell in vitro. BMC Cell Biol 7: 14, 2006.

16. Wagner W, Horn P, Castoldi M, Diehlmann A, Bork S, Saffrich R, Benes V, Blake J, Pfister S, Eckstein V and Ho AD: Replicative senescence of mesenchymal stem cells: A continuous and organized process. PLoS One 3: e2213, 2008.

17. Bakopoulou A, Leyhausen G, Volk J, Tsiftsoglou A, Garefis P, Koidis P and Geurtsen W: Assessment of the impact of two different isolation methods on the osteo/odontogenic differentiation potential of human dental stem cells derived from deciduous teeth. Calcif Tissue Int 88: 130-141, 2011.

18. Naderi N, Wilde C, Haque T, Francis W, Seifalian AM, Thornton CA, Xia Z and Whitaker IS: Adipogenic differentiation of adipose-derived stem cells in 3-dimensional spheroid cultures (microtissue): Implications for the reconstructive surgeon. J Plast Reconstr Aesthet Surg 67: 1726-1734, 2014.
19. Bakopoulou A, Leyhausen G, Volk J, Tsiftsoglou A, Garefis P, Koidis P and Geurtsen W: Comparative analysis of in vitro osteo/odontogenic differentiation potential of human dental pulp stem cells (DPSCs) and stem cells from the apical papilla (SCAP). Arch Oral Biol 56: 709-721, 2011.

20. Livak KJ and Schmittgen TD: Analysis of relative gene expression data using real-time quantitative PCR and the 2(-Delta Delta $\mathrm{C}(\mathrm{T})$ ) method. Methods 25: 402-408, 2001.

21. Dimri GP, Lee X, Basile G, Acosta M, Scott G, Roskelley C, Medrano EE, Linskens M, Rubelj I, Pereira-Smith O, et al: A biomarker that identifies senescent human cells in culture and in aging skin in vivo. Proc Natl Acad Sci USA 92: 9363-9367, 1995.

22. Childs BG, Durik M, Baker DJ and van Deursen JM: Cellular senescence in aging and age-related disease: From mechanisms to therapy. Nat Med 21: 1424-1435, 2015.

23. Dong CM, Wang XL, Wang GM, Zhang WJ, Zhu L, Gao S, Yang DJ, Qin Y, Liang QJ, Chen YL, et al: A stress-induced cellular aging model with postnatal neural stem cells. Cell Death Dis 5: e1116, 2014.

24. Estrada JC, Torres Y, Benguría A, Dopazo A, Roche E, Carrera-Quintanar L, Pérez RA, Enríquez JA, Torres R, Ramírez JC, et al: Human mesenchymal stem cell-replicative senescence and oxidative stress are closely linked to aneuploidy. Cell Death Dis 4: e691, 2013.

25. Hayflick L: The Limited in vitro lifetime of human diploid cell strains. Exp Cell Res 37: 614-636, 1965.

26. Li Z, Liu C, Xie Z, Song P, Zhao RC, Guo L, Liu Z and Wu Y: Epigenetic dysregulation in mesenchymal stem cell aging and spontaneous differentiation. PLoS One 6: e20526, 2011.

27. $\mathrm{Wu} \mathrm{Y}$ and Zhao RC: The role of chemokines in mesenchymal stem cell homing to myocardium. Stem Cell Rev 8: 243-250, 2012.

28. Philippe B, Luc S, Valerie PB, Jerome R, Alessandra BR and Louis C: Culture and use of mesenchymal stromal cells in phase I and II clinical trials. Stem Cells Int 2010: 503593, 2010.

29. Suchánek J, Visek B, Soukup T, El-Din Mohamed SK, Ivancaková R, Mokrỳ J, Aboul-Ezz EH and Omran A: Stem cells from human exfoliated deciduous teeth-isolation, long term cultivation and phenotypical analysis. Acta Medica (Hradec Kralove) 53: 93-99, 2010

30. Madeira A, da Silva CL, dos Santos F, Camafeita E, Cabral JM and Sa-Correia I: Human mesenchymal stem cell expression program upon extended ex-vivo cultivation, as revealed by 2-DE-based quantitative proteomics. PLoS One 7: e43523, 2012.

31. Andersen RK, Zaher W, Larsen KH, Ditzel N, Drews K, Wruck W, Adjaye J, Abdallah BM and Kassem M: Association between in vivo bone formation and ex vivo migratory capacity of human bone marrow stromal cells. Stem Cell Res Ther 6: 196, 2015.

32. Kuilman T, Michaloglou C, Mooi WJ and Peeper DS: The essence of senescence. Genes Dev 24: 2463-2479, 2010.

33. van Deursen JM: The role of senescent cells in ageing. Nature 509: 439-446, 2014

34. Ksiazek K: A comprehensive review on mesenchymal stem cell growth and senescence. Rejuvenation Res 12: 105-116, 2009.

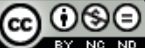

This work is licensed under a Creative Commons Attribution-NonCommercial-NoDerivatives 4.0 International (CC BY-NC-ND 4.0) License. 